\title{
Universal Learning Theory
}

\author{
Marcus Hutter \\ RSISE@ANU and SML @ NICTA \\ Canberra, ACT, 0200, Australia \\ marcus@hutter1.net ww.hutter1.net
}

February 2011

\begin{abstract}
This encyclopedic article gives a mini-introduction into the theory of universal learning, founded by Ray Solomonoff in the 1960s and significantly developed and extended in the last decade. It explains the spirit of universal learning, but necessarily glosses over technical subtleties.
\end{abstract}

\section{Contents}

1 Definition, Motivation and Background

2 Deterministic Environments

3 Algorithmic Probability

4 Universal Bayes

5 Applications

6 Discussion and Future Directions

Recommended Reading

\section{Keywords}

Algorithmic probability; Ray Solomonoff; induction; prediction; decision; action; Turing machine; Kolmogorov complexity; universal prior; Bayes' rule. 


\section{Definition, Motivation and Background}

Universal (machine) learning is concerned with the development and study of algorithms that are able to learn from data in a very large range of environments with as few assumptions as possible. The class of environments typically considered includes all computable stochastic processes. The investigated learning tasks range from inductive inference, sequence prediction, sequential decisions, to (re)active problems like reinforcement learning [Hut05], but also include clustering, regression, and others [LV08]. Despite various no-free-lunch theorems [WM97], universal learning is possible by assuming that the data possess some effective structure, but without specifying any further, which structure. Learning algorithms that are universal (at least to some degree) are also necessary for developing autonomous general intelligent systems, required e.g. for exploring other planets, as opposed to decision support systems which keep a human in the loop. There is also an intrinsic interest in striving for generality: Finding new learning algorithms for every particular (new) problem is possible but cumbersome and prone to disagreement or contradiction. A sound formal general and ideally complete theory of learning can unify existing approaches, guide the development of practical learning algorithms, and last but not least lead to novel and deep insights.

This encyclopedic article gives a mini-introduction into the theory of universal learning, founded by Ray Solomonoff in the 1960s [Sol64, Sol78] and significantly developed and extended by the author and his colleagues in the last decade. It is based on [Hut05]. It explains the spirit of universal learning, but necessarily glosses over many technical subtleties. Precise formulation of the results with proofs and/or references to original publications can be found in [Hut05].

\section{Deterministic Environments}

Let $t, n \in \mathbb{N}$ be natural numbers, $\mathcal{X}^{*}$ be the set of finite strings and $\mathcal{X}^{\infty}$ be the set of infinite sequences over some alphabet $\mathcal{X}$ of size $|\mathcal{X}|$. For a string $x \in \mathcal{X}^{*}$ of length $\ell(x)=n$ we write $x_{1} x_{2} \ldots x_{n}$ with $x_{t} \in \mathcal{X}$, and further abbreviate $x_{t: n}:=x_{t} x_{t+1} \ldots x_{n-1} x_{n}$ and $x_{<n}:=x_{1} \ldots x_{n-1}$, and $\epsilon=x_{<1}$ for the empty string. Consider a countable class of hypotheses $\mathcal{M}=\left\{H_{1}, H_{2}, \ldots\right\}$. Each hypothesis $H \in \mathcal{M}$ (also called model) shall describe an infinite sequence $x_{1: \infty}^{H}$, e.g. like in IQ test questions " $2,4,6,8, \ldots$. ". In online learning, for $t=1,2,3, \ldots$, we predict $x_{t}$ based on past observations $\dot{x}_{<t}$, then nature reveals $\dot{x}_{t}$, and so on, where the dot above $x$ indicates the true observation. We assume that the true hypothesis is in $\mathcal{M}$, i.e. $\dot{x}_{1: \infty}=x_{1: \infty}^{H_{m}}$ for some $m \in \mathbb{N}$. Goal is to ("quickly") identify the unknown $H_{m}$ from the observations.

Learning by enumeration works as follows: Let $\mathcal{M}_{t}=\left\{H \in \mathcal{M}: x_{<t}^{H}=\dot{x}_{<t}\right\}$ be the set of hypotheses consistent with our observations $\dot{x}_{<t}$ so far. The hypothesis in $\mathcal{M}_{t}$ with smallest index, say $m_{t}^{\prime}$, is selected and used for predicting $x_{t}$. Then $\dot{x}_{t}$ is observed and all $H \in \mathcal{M}_{t}$ inconsistent with $x_{t}$ are eliminated, i.e. they are not 
included in $\mathcal{M}_{t+1}$. Every prediction error results in the elimination of at least $H_{m_{t}^{\prime}}$, so after at most $m-1$ errors, the true hypothesis $H_{m}$ gets selected forever, since it never makes an error $\left(H_{m} \in \mathcal{M}_{t} \forall t\right)$. This identification may take arbitrarily long (in $t$ ), but the number of errors on the way is bounded by $m-1$, and the latter is often more important. As an example for which the bound is attained, consider $H_{i}$ with $x_{1: \infty}^{H_{i}}:=1^{f(i)} 0^{\infty} \forall i$ for any strictly increasing function $f$, e.g. $f(i)=i$. But we now show that we can do much better than this, at least for finite $\mathcal{X}$.

Majority learning. Consider (temporarily in this paragraph only) a binary alphabet $\mathcal{X}=\{0,1\}$ and a finite deterministic hypothesis class $\mathcal{M}=\left\{H_{1}, H_{2}, \ldots, H_{N}\right\} . H_{m}$ and $\mathcal{M}_{t}$ are as before, but now we take a majority vote among the hypotheses in $\mathcal{M}_{t}$ as our prediction of $x_{t}$. If the prediction turns out to be wrong, then at least half (the majority) of the hypotheses get eliminated from $\mathcal{M}_{t}$. Hence after at most $\log N$ errors, there is only a single hypothesis, namely $H_{m}$, left over. So this majority predictor makes at most $\log N$ errors. As an example where this bound is essentially attained, consider $m=N=2^{n}-1$ and let $x_{1: \infty}^{H_{i}}$ be the digits after the comma of the binary expansion of $(i-1) / 2^{n}$ for $i=1, \ldots, N$.

Weighted majority for countable classes. Majority learning can be adapted to denumerable classes $\mathcal{M}$ and general finite alphabet $\mathcal{X}$ as follows: Each hypothesis $H_{i}$ is assigned a weight $w_{i}>0$ with $\sum_{i} w_{i} \leq 1$. Let $W:=\sum_{i: H_{i} \in \mathcal{M}_{t}} w_{i}$ be the total weight of the hypotheses in $\mathcal{M}_{t}$. Let $\mathcal{M}_{t}^{a}:=\left\{H_{i} \in \mathcal{M}_{t}: x_{t}^{H_{i}}=a\right\}$ be the consistent hypotheses predicting $x_{t}=a$, and $W_{a}$ their weight, and take the weighted majority prediction $x_{t}=\operatorname{argmax}_{a} W_{a}$. Similarly as above, a prediction error decreases $W$ by a factor of $1-1 /|\mathcal{X}|$, since $\max _{a} W_{a} \geq W /|\mathcal{X}|$. Since $w_{m} \leq W \leq 1$, this algorithm can at most make $\log _{1-1 /|\mathcal{X}|} w_{m}=O\left(\log w_{m}^{-1}\right)$ prediction errors. If we choose for instance $w_{i}=(i+1)^{-2}$, the number of errors is $O(\log m)$, which is an exponential improvement over the Gold-style learning by enumeration above.

\section{Algorithmic Probability}

Algorithmic probability has been founded by Solomonoff [Sol64]. The so-called universal probability or a-priori probability is the key quantity for universal learning. Its philosophical and technical roots are Ockham's razor (choose the simplest model consistent with the data), Epicurus' principle of multiple explanations (keep all explanations consistent with the data), (Universal) Turing machines (to compute, quantify and assign codes to all quantities of interest), and Kolmogorov complexity (to define what simplicity/complexity means). This section considers deterministic computable sequences, and the next section the general setup of computable probability distributions.

(Universal) monotone Turing machines. Since we consider infinite computable sequences, we need devices that convert input data streams to output data streams. For this we define the following variants of a classical deterministic Turing Machine: 
A monotone Turing machine $T$ is defined as a Turing machine with one unidirectional input tape, one unidirectional output tape, and some bidirectional work tapes. The input tape is binary (no blank) and read only, the output tape is over finite alphabet $\mathcal{X}$ (no blank) and write only, unidirectional tapes are those where the head can only move from left to right, work tapes are initially filled with zeros and the output tape with some fixed element from $\mathcal{X}$. We say that monotone Turing machine $T$ outputs/computes a string starting with $x$ on input $p$, and write $T(p)=x *$ if $p$ is to the left of the input head when the last bit of $x$ is output ( $T$ reads all of $p$ but no more). $T$ may continue operation and need not halt. For a given $x$, the set of such $p$ forms a prefix code. Such codes are called minimal programs. Similarly we write $T(p)=\omega$ if $p$ outputs the infinite sequence $\omega$. A prefix code $\mathcal{P}$ is a set of binary strings such that no element is proper prefix of another. It satisfies Kraft's inequality $\sum_{p \in \mathcal{P}} 2^{-\ell(p)} \leq 1$.

The table of rules of a Turing machine $T$ can be prefix encoded in a canonical way as a binary string, denoted by $\langle T\rangle$. Hence, the set of Turing machines $\left\{T_{1}, T_{2}, \ldots\right\}$ can be effectively enumerated. There are so-called universal Turing machines that can "simulate" all other Turing machines. We define a particular one which simulates monotone Turing machine $T(q)$ if fed with input $\langle T\rangle q$, i.e. $U(\langle T\rangle q)=T(q) \forall T, q$. Note that for $p$ not of the form $\langle T\rangle q, U(p)$ does not output anything. We call this particular $U$ the reference universal Turing machine.

Universal weighted majority learning. $T_{1}(\epsilon), T_{2}(\epsilon), \ldots$ constitutes an effective enumeration of all finite and infinite computable sequences, hence also monotone $U(p)$ for $p \in\{0,1\}^{*}$. As argued below, the class of computable infinite sequences, is conceptually very interesting. The halting problem implies that there is no recursive enumeration of all partial-recursive functions with infinite domain; hence we cannot remove the finite sequences algorithmically. It is very fortunate that we don't have to. Hypothesis $H_{p}$ is identified with the sequence $U(p)$, which may be finite, infinite, or possibly even empty. The class of considered hypotheses is $\mathcal{M}:=\left\{H_{p}: p \in\{0,1\}^{*}\right\}$.

The weighted majority algorithm also needs weights $w_{p}$ for each $H_{p}$. Ockham's razor combined with Epicurus' principle demand to assign a high (low) prior weight to a simple (complex) hypothesis. If complexity is identified with program length, then $w_{p}$ should be a decreasing function of $\ell(p)$. It turns out that $w_{p}=2^{-\ell(p)}$ is the "right" choice, since minimal $p$ form a prefix code and therefore $\sum_{p} w_{p} \leq 1$ as required.

Using $H_{p}$ for prediction can now fail in two ways. $H_{p}$ may make a wrong prediction or no prediction at all for $x_{t}$. The true hypothesis $H_{m}$ is still assumed to produce an infinite sequence. The weighted majority algorithm in this setting makes at most $O\left(\log w_{p}^{-1}\right)=O(\ell(p))$ errors. It is also plausible that learning $\ell(p)$ bits requires $O(\ell(p))$ "trials".

Universal mixture prediction. Solomonoff [Sol78] defined the following universal a-priori probability

$$
M(x):=\sum_{p: U(p)=x *} 2^{-\ell(p)}
$$


That is, $M(x)=W$ is the total weight of the computable deterministic hypotheses consistent with $x$ for the universal weight choice $w_{p}=2^{-\ell(p)}$. The universal weighted majority algorithm predicted $\operatorname{argmax}_{a} M\left(\dot{x}_{<t} a\right)$. Instead, one could also make a probability prediction $M\left(a \mid \dot{x}_{<t}\right):=M\left(\dot{x}_{<t} a\right) / M\left(\dot{x}_{<t}\right)$, which is the relative weight of hypotheses in $\mathcal{M}_{t}$ predicting $a$. The higher the probability $M\left(\dot{x}_{t} \mid \dot{x}_{<t}\right)$ assigned to the true next observation $\dot{x}_{t}$, the better. Consider the absolute prediction error $\mid 1-$ $M\left(\dot{x}_{t} \mid \dot{x}_{<t}\right) \mid$ and the logarithmic error $-\log M\left(\dot{x}_{t} \mid \dot{x}_{<t}\right)$. The cumulative logarithmic error is bounded by $\sum_{t=1}^{n}-\log M\left(\dot{x}_{t} \mid \dot{x}_{<t}\right)=-\log M\left(\dot{x}_{1: n}\right) \leq \ell(p)$ for any program $p$ that prints $\dot{x} *$. For instance $p$ could be chosen as the shortest one printing $\dot{x}_{1: \infty}$, which has length $\operatorname{Km}\left(\dot{x}_{1: \infty}\right):=\min \left\{\ell(p): U(p)=\dot{x}_{1: \infty}\right\}$. Using $1-z \leq-\log z$ and letting $n \rightarrow \infty$ we get

$$
\sum_{t=1}^{\infty}\left|1-M\left(\dot{x}_{t} \mid \dot{x}_{<t}\right)\right| \leq \sum_{t=1}^{\infty}-\log M\left(\dot{x}_{t} \mid \dot{x}_{<t}\right) \leq K m\left(\dot{x}_{1: \infty}\right)
$$

Hence again, the cumulative absolute and logarithmic errors are bounded by the number of bits required to describe the true environment.

\section{Universal Bayes}

The exposition so far has dealt with deterministic environments only. Data sequences produced by real-world processes are rarely as clean as IQ test sequences. They are often noisy. This section deals with stochastic sequences sampled from computable probability distributions. The developed theory can be regarded as an instantiation of Bayesian learning. Bayes' theorem allows to update beliefs in face of new information but is mute about how to choose the prior and the model class to begin with. Subjective choices based on prior knowledge are informal, and traditional 'objective' choices like Jeffrey's prior are not universal. Machine learning, the computer science branch of statistics, develops (fully) automatic inference and decision algorithms for very large problems. Naturally, machine learning has (re)discovered and exploited different principles (Ockham's and Epicurus') for choosing priors, appropriate for this situation. This leads to an alternative representation of universal probability as a mixture over all lower semi-computable semimeasures with Kolmogorov complexity based prior as described below.

Bayes. Sequences $\omega=\omega_{1: \infty} \in \mathcal{X}^{\infty}$ are now assumed to be sampled from the "true" probability measure $\mu$, i.e. $\mu\left(x_{1: n}\right):=\mathrm{P}\left[\omega_{1: n}=x_{1: n} \mid \mu\right]$ is the $\mu$-probability that $\omega$ starts with $x_{1: n}$. Expectations w.r.t. $\mu$ are denoted by $\mathbf{E}$. In particular for a function $f: \mathcal{X}^{n} \rightarrow \mathbb{R}$, we have $\mathbf{E}[f]=\mathbf{E}\left[f\left(\omega_{1: n}\right)\right]=\sum_{x_{1: n}} \mu\left(x_{1: n}\right) f\left(x_{1: n}\right)$. Note that in Bayesian learning, measures, environments, and models are the same objects; let $\mathcal{M}=\left\{\nu_{1}, \nu_{2}, \ldots\right\} \equiv\left\{H_{\nu_{1}}, H_{\nu_{2}}, \ldots\right\}$ denote a countable class of these measures $\equiv$ hypotheses. Assume that $\mu$ is unknown but known to be a member of 
$\mathcal{M}$, and $w_{\nu}:=\mathrm{P}\left[H_{\nu}\right]$ is the given prior belief in $H_{\nu}$. Then the Bayes mixture

$$
\xi\left(x_{1: n}\right):=\mathrm{P}\left[\omega_{1: n}=x_{1: n}\right]=\sum_{\nu \in \mathcal{M}} \mathrm{P}\left[\omega_{1: n}=x_{1: n} \mid H_{\nu}\right] \mathrm{P}\left[H_{\nu}\right] \equiv \sum_{\nu \in \mathcal{M}} \nu\left(x_{1: n}\right) w_{\nu}
$$

must be our a-priori belief in $x_{1: n}$, and $\mathrm{P}\left[H_{\nu} \mid \omega_{1: n}=x_{1: n}\right]=w_{\nu} \nu\left(x_{1: n}\right) / \xi\left(x_{1: n}\right)$ be our posterior belief in $\nu$ by Bayes' rule.

Universal choice of $\mathcal{M}$. Next we need to find a universal class of environments $\mathcal{M}_{U}$. Roughly speaking, Bayes works if $\mathcal{M}$ contains the true environment $\mu$. The larger $\mathcal{M}$ the less restrictive is this assumption. The class of all computable distributions, although only countable, is pretty large from a practical point of view, since it includes for instance all of today's valid physics theories. (Finding a non-computable physical system would indeed overturn the generally accepted Church-Turing thesis.) It is the largest class, relevant from a computational point of view. Solomonoff Sol64, Eq.(13)] defined and studied the mixture over this class.

One problem is that this class is not (effectively=recursively) enumerable, since the class of computable functions is not enumerable due to the halting problem, nor is it decidable whether a function is a measure. Hence $\xi$ is completely incomputable. Leonid Levin [ZL70] had the idea to "slightly" extend the class and include also lower semi-computable semimeasures.

A function $\nu: \mathcal{X}^{*} \rightarrow[0,1]$ is a called a semimeasure iff $\nu(x) \geq \sum_{a \in \mathcal{X}} \nu(x a) \forall x \in \mathcal{X}^{*}$. It is a proper probability measure iff equality holds and $\nu(\epsilon)=1 . \nu(x)$ still denotes the $\nu$-probability that a sequence starts with string $x$. A function is called lower semi-computable, if it can be approximated from below. Similarly to that fact that the class of partial recursive functions is recursively enumerable, one can show that the class $\mathcal{M}_{U}=\left\{\nu_{1}, \nu_{2}, \ldots\right\}$ of lower semi-computable semimeasures is recursively enumerable. In some sense $\mathcal{M}_{U}$ is the largest class of environments for which $\xi$ is in some sense computable, but even larger classes are possible [Sch02].

Kolmogorov complexity. Before we can turn to the prior $w_{\nu}$, we need to quantify complexity/simplicity. Intuitively, a string is simple if it can be described in a few words, like "the string of one million ones", and is complex if there is no such short description, like for a random object whose shortest description is specifying it bit by bit. We are interested in effective descriptions, and hence restrict decoders to be Turing machines. One can define the prefix Kolmogorov complexity of string $x$ as the length $\ell$ of the shortest halting program $p$ for which $U$ outputs $x$ :

$$
K(x):=\min _{p}\{\ell(p): U(p)=x \text { halts }\}
$$

Simple strings like $000 \ldots 0$ can be generated by short programs, and, hence have low Kolmogorov complexity, but irregular (e.g. random) strings are their own shortest description, and hence have high Kolmogorov complexity. For non-string objects $o$ (like numbers and functions) one defines $K(o):=K(\langle o\rangle)$, where $\langle o\rangle \in \mathcal{X}^{*}$ is some standard code for $o$. In particular, $K\left(\nu_{i}\right)=K(i)$. 
To be brief, $K$ is an excellent universal complexity measure, suitable for quantifying Ockham's razor.

The universal prior. We can now quantify a prior biased towards simple models. First, we quantify the complexity of an environment $\nu$ or hypothesis $H_{\nu}$ by its Kolmogorov complexity $K(\nu)$. The universal prior should be a decreasing function in the model's complexity, and of course sum to (less than) one. Since $\sum_{x} 2^{-K(x)} \leq 1$ by the prefix property and Kraft's inequality, this suggests the choice

$$
w_{\nu}=w_{\nu}^{U}:=2^{-K(\nu)}
$$

Since $\log i \leq K\left(\nu_{i}\right) \leq \log i+2 \log \log i$ for "most" $i$, most $\nu_{i}$ have prior approximately reciprocal to their index $i$ as also advocated by Jeffreys and Rissanen.

Representations. Combining the universal class $\mathcal{M}_{U}$ with the universal prior (21), we arrive at the universal mixture

$$
\xi_{U}(x):=\sum_{\nu \in \mathcal{M}_{U}} 2^{-K(\nu)} \nu(x)
$$

which has remarkable properties. First, it is itself a lower semi-computable semimeasure, that is $\xi_{U} \in \mathcal{M}_{U}$, which is very convenient. Note that for most classes, $\xi \notin \mathcal{M}$.

Second, $\xi_{U}$ coincides with $M$ within an irrelevant multiplicative constant, and $M \in \mathcal{M}_{U}$. This means that the mixture over deterministic computable sequences is as rich as the mixture over the much larger class of semi-computable semimeasures. The intuitive reason is that the probabilistic semimeasures are in the convex hull of the deterministic ones, and therefore need not be taken extra into account in the mixture.

There is another, possibly the simplest, representation: One can show that $M(x)$ is equal to the probability that $U$ outputs a string starting with $x$ when provided with uniform random noise on the program tape. Note that a uniform distribution is also used in many no-free-lunch theorems to prove the impossibility of universal learners, but in our case the uniform distribution is piped through a universal Turing machine, which defeats these negative implications as we will see in the next section.

\section{Applications}

In the stochastic case, identification of the true hypothesis is problematic. The posterior $\mathrm{P}[H \mid x]$ may not concentrate around the true hypothesis $H_{\mu}$ if there are other hypotheses $H_{\nu}$ that are not asymptotically distinguishable from $H_{\mu}$. But even if model identification (induction in the narrow sense) fails, predictions, decisions, and actions can be good, and indeed, for universal learning this is generally the case.

Universal sequence prediction. Given a sequence $x_{1} x_{2} \ldots x_{t-1}$, we want to predict its likely continuation $x_{t}$. We assume that the strings which have to be continued 
are drawn from a computable "true" probability distribution $\mu$. The maximal prior information a prediction algorithm can possess is the exact knowledge of $\mu$, but often the true distribution is unknown. Instead, prediction is based on a guess $\rho$ of $\mu$. Let $\rho(a \mid x):=\rho(x a) / \rho(x)$ be the "predictive" $\rho$-probability that the next symbol is $a \in \mathcal{X}$, given sequence $x \in \mathcal{X}^{*}$. Since $\mu \in \mathcal{M}_{U}$ it is natural to use $\xi_{U}$ or $M$ for prediction.

Solomonoff's [Sol78, Hut05] celebrated result indeed shows that $M$ converges to $\mu$. For general alphabet it reads

$$
\sum_{t=1}^{\infty} \mathbf{E}\left[\sum_{a \in \mathcal{X}}\left(M\left(a \mid \omega_{<t}\right)-\mu\left(a \mid \omega_{<t}\right)\right)^{2}\right] \leq K(\mu) \ln 2+O(1)
$$

Analogous bounds hold for $\xi_{U}$ and for other than the Euclidian distance, e.g. the Hellinger and the absolute distance and the relative entropy.

For a sequence $a_{1}, a_{2}, \ldots$ of random variables, $\sum_{t=1}^{\infty} \mathbf{E}\left[a_{t}^{2}\right] \leq c<\infty$ implies $a_{t} \rightarrow 0$ for $t \rightarrow \infty$ with $\mu$-probability 1 (w.p.1). Convergence is rapid in the sense that the probability that $a_{t}^{2}$ exceeds $\varepsilon>0$ at more than $c / \varepsilon \delta$ times, is bounded by $\delta$. This might loosely be called the number of errors. Hence Solomonoff's bounds implies

$$
M\left(x_{t} \mid \omega_{<t}\right)-\mu\left(x_{t} \mid \omega_{<t}\right) \longrightarrow 0 \text { for any } x_{t} \text { rapid w.p.1 for } t \rightarrow \infty
$$

The number of times, $M$ deviates from $\mu$ by more than $\varepsilon>0$ is bounded by $O(K(\mu))$, i.e. is proportional to the complexity of the environment, which is again reasonable. A counting argument shows that $O(K(\mu))$ errors for most $\mu$ are unavoidable. No other choice for $w_{\nu}$ would lead to significantly better bounds. Again, in general it is not possible to determine when these "errors" occur. Multi-step lookahead convergence $M\left(x_{t: n_{t}} \mid \omega_{<t}\right)-\mu\left(x_{t: n_{t}} \mid \omega_{<t}\right) \rightarrow 0$ even for unbounded lookahead $n_{t}-t \geq 0$, relevant for delayed sequence prediction and in reactive environments, can also be shown.

In summary, $M$ is an excellent sequence predictor under the only assumption that the observed sequence is drawn from some (unknown) computable probability distribution. No ergodicity, stationarity, or identifiability or other assumption is required.

Universal sequential decisions. Predictions usually form the basis for decisions and actions, which result in some profit or loss. Let $\ell_{x_{t} y_{t}} \in[0,1]$ be the received loss for decision $y_{t} \in \mathcal{Y}$ when $x_{t} \in \mathcal{X}$ turns out to be the true $t^{t h}$ symbol of the sequence. The $\rho$-optimal strategy

$$
y_{t}^{\Lambda_{\rho}}\left(\omega_{<t}\right):=\arg \min _{y_{t}} \sum_{x_{t}} \rho\left(x_{t} \mid \omega_{<t}\right) \ell_{x_{t} y_{t}}
$$

minimizes the $\rho$-expected loss. For instance, if we can decide among $\mathcal{Y}=\{$ sunglasses, umbrella $\}$ and it turns out to be $\mathcal{X}=\{$ sun,rain $\}$, and our personal loss matrix is $\ell=\left(\begin{array}{ll}0.0 & 0.1 \\ 1.0 & 0.3\end{array}\right)$, then $\Lambda_{\rho}$ takes $y_{t}^{\Lambda_{\rho}}=$ sunglasses if $\rho\left(\right.$ rain $\left.\mid \omega_{<t}\right)<1 / 8$ and an umbrella otherwise. For $\mathcal{X}=\mathcal{Y}$ and $0-1$ loss $\ell_{x y}=0$ for $x=y$ and 1 else, $\Lambda_{\rho}$ predicts the most likely symbol $y_{t}^{\Lambda_{\rho}}=\operatorname{argmax}_{a} \rho\left(a \mid \omega_{<t}\right)$ as in Section 2 . 
The cumulative $\mu$ (=true)-expected loss of $\Lambda_{\rho}$ for the first $n$ symbols is

$$
\operatorname{Loss}_{n}^{\Lambda_{\rho}}:=\sum_{t=1}^{n} \mathbf{E}\left[\ell_{\omega_{t} y_{t}^{\Lambda} \rho\left(\omega_{<t}\right)}\right] \equiv \sum_{t=1}^{n} \sum_{x_{1: t}} \mu\left(x_{1: t}\right) \ell_{x_{t} y_{t}^{\Lambda} \rho\left(x_{<t}\right)}
$$

If $\mu$ is known, $\Lambda_{\mu}$ obviously results in the best decisions in the sense of achieving minimal expected loss among all strategies. For the predictor $\Lambda_{M}$ based on $M$ (and similarly $\left.\xi_{U}\right)$, one can show

$$
\sqrt{\operatorname{Loss}_{n}^{\Lambda_{M}}}-\sqrt{\operatorname{Loss}_{n}^{\Lambda_{\mu}}} \leq \sqrt{2 K(\mu) \ln 2+O(1)}
$$

This implies that $\operatorname{Loss}_{n}^{\Lambda_{M}} / \operatorname{Loss}_{n}^{\Lambda_{\mu}} \rightarrow 1$ for $\operatorname{Loss}_{n}^{\Lambda_{\mu}} \rightarrow \infty$, or if $\operatorname{Loss}_{\infty}^{\Lambda_{\mu}}$ is finite, then also $\operatorname{Loss}_{\infty}^{\Lambda_{M}}<\infty$. This shows that $M$ (via $\Lambda_{M}$ ) performs also excellent from a decision-theoretic perspective, i.e. suffers loss only slightly larger than the optimal $\Lambda_{\mu}$ strategy.

One can also show that $\Lambda_{M}$ is Pareto-optimal (admissible) in the sense that every other predictor with smaller loss than $\Lambda_{M}$ in some environment $\nu \in \mathcal{M}_{U}$ must be worse in another environment.

Universal classification and regression. The goal of classification and regression is to infer the functional relationship $f: \mathcal{Y} \rightarrow \mathcal{X}$ from data $\left\{\left(y_{1}, x_{1}\right), \ldots,\left(y_{n}, x_{n}\right)\right\}$. In a predictive online setting one wants to "directly" infer $x_{t}$ from $y_{t}$ given $\left(y_{<t}, x_{<t}\right)$ for $t=1,2,3, \ldots$. The universal induction framework has to be extended by regarding $y_{1: \infty}$ as independent side-information presented in form of an oracle or extra tape information or extra parameter. The construction has to ensure that $x_{1: n}$ only depends on $y_{1: n}$ but is (functionally or statistically) independent of $y_{n+1: \infty}$.

First, we augment a monotone Turing machine with an extra input tape containing $y_{1: \infty}$. The Turing machine is called chronological if it does not read beyond $y_{1: n}$ before $x_{1: n}$ has been written. Second, semimeasures $\rho=\mu, \nu, M, \xi_{U}$ are extended to $\rho\left(x_{1: n} \mid y_{1: \infty}\right)$, i.e. one semimeasure $\rho\left(\cdot \mid y_{1: \infty}\right)$ for each $y_{1: \infty}$ (no distribution over $y$ is assumed). Any such semimeasure must be chronological in the sense that $\rho\left(x_{1: n} \mid y_{1: \infty}\right)$ is independent of $y_{t}$ for $t>n$, hence we can write $\rho\left(x_{1: n} \mid y_{1: n}\right)$. In classification and regression, $\rho$ is typically (conditionally) i.i.d., i.e. $\rho\left(x_{1: n} \mid y_{1: n}\right)=\prod_{t=1}^{n} \rho\left(x_{t} \mid y_{t}\right)$, which is chronological, but note that the Bayes mixture $\xi$ is not i.i.d. One can show that the class of lower semi-computable chronological semimeasures $\mathcal{M}_{U}^{\mid}=\left\{\nu_{1}(\cdot \mid \cdot), \nu_{2}(\cdot \mid \cdot), \ldots\right\}$ is effectively enumerable.

The generalized universal a-priori semimeasure also has two equivalent definitions:

$$
M\left(x_{1: n} \mid y_{1: n}\right):=\sum_{p: U\left(p, y_{1: n}\right)=x_{1: n}} 2^{-\ell(p)}=\sum_{\nu \in \mathcal{M}} 2^{-K(\nu)} \nu\left(x_{1: n} \mid y_{1: n}\right)
$$

which is again in $\mathcal{M}_{U}^{\mid}$. In case of $|\mathcal{Y}|=1$, this reduces to (1) and (3) . The bounds (4) and (5) and others continue to hold, now for all individual $y$ 's, i.e. $M$ predicts asymptotically $x_{t}$ from $y_{t}$ and $\left(y_{<t}, x_{<t}\right)$ for any $y$, provided $x$ is sampled from a 
computable probability measure $\mu\left(\cdot \mid y_{1: \infty}\right)$. Convergence is rapid if $\mu$ is not too complex.

Universal reinforcement learning. The generalized universal a-priori semimeasure (6) can be used to construct a universal reinforcement learning agent, called AIXI. In reinforcement learning, an agent interacts with an environment in cycles $t=1,2, \ldots, n$. In cycle $t$, the agent chooses an action $y_{t}$ (e.g. a limb movement) based on past perceptions $x_{<t}$ and past actions $y_{<t}$. Thereafter, the agent perceives $x_{t} \equiv o_{t} r_{t}$, which consists of a (regular) observation $o_{t}$ (e.g. a camera image) and a real-valued reward $r_{t}$. The reward may be scarce, e.g. just $+1(-1)$ for winning (losing) a chess game, and 0 at all other times. Then the next cycle $t+1$ starts. The goal of the agent is to maximize its expected reward over its lifetime $n$. Probabilistic planning deals with the situation in which the environmental probability distribution $\mu\left(x_{1: n} \mid y_{1: n}\right)$ is known. Reinforcement learning deals with the case of unknown $\mu$. In universal reinforcement learning, the unknown $\mu$ is replaced by $M$ similarly to the prediction, decision, and classification cases above. The universally optimal action in cycle $t$ is Hut05

$$
y_{t}:=\arg \max _{y_{t}} \sum_{x_{t}} \ldots \max _{y_{n}} \sum_{x_{n}}\left[r_{t}+\ldots+r_{n}\right] M\left(x_{1: n} \mid y_{1: n}\right)
$$

The expectations $(\Sigma)$ and maximizations $(\max )$ over future $x$ and $y$ are interleaved in chronological order to form an expectimax tree similarly to minimax decision trees in extensive zero-sum games like chess. Optimality and universality results similar to the prediction case exist.

Approximations and practical applications. Since $K$ and $M$ are only semicomputable, they have to be approximated in practice. For instance, $-\log M(x)=$ $K(x)+O(\log \ell(x))$, and $K(x)$ can and has been approximated by off-the-shelf compressors like Lempel-Ziv and successfully applied to a plethora of clustering problems CV05. The approximations upper-bound $K(x)$ and e.g. for Lempel-Ziv converge to $K(x)$ if $x$ is sampled from a context tree source. The Minimum Description Length principle [Grü07] also attempts to approximate $K(x)$ for stochastic $x$. The Context Tree Weighting algorithm considers a relatively large subclass of $\mathcal{M}_{U}$ that can be summed over efficiently. This can and has been combined with Monte-Carlo sampling to efficiently approximate AIXI (7) [VNHS10]. The time-bounded versions of $K$ and $M$, namely Levin complexity $K t$ and the speed prior $S$ have also been applied to various learning tasks Gag07.

Other applications. Continuously parameterized model classes are very common in statistics. Bayesian's usually assume a prior density over some parameter $\theta \in \mathbb{R}^{d}$, which works fine for many problems, but has its problems. Even for continuous classes $\mathcal{M}$, one can assign a (proper) universal prior (not density) $w_{\theta}^{U}:=2^{-K(\theta)}>0$ for computable $\theta$ (and $\nu_{\theta}$ ), and 0 for uncomputable ones. This effectively reduces $\mathcal{M}$ to a discrete class $\left\{\nu_{\theta} \in \mathcal{M}: w_{\theta}^{U}>0\right\} \subseteq \mathcal{M}_{U}$ which is typically dense in $\mathcal{M}$. There are various fundamental philosophical and statistical problems and paradoxes around 
(Bayesian) induction, which nicely disappear in the universal framework. For instance, universal induction has no zero and no improper $\mathrm{p}$ (oste)rior problem, i.e. can confirm universally quantified hypotheses, is reparametrization and representation invariant, and avoids the old-evidence and updating problem, in contrast to most classical continuous prior densities. It even performs well in incomputable environments, actually better than latter [Hut07.

\section{Discussion and Future Directions}

Universal learning is designed to work for a wide range of problems without any apriori knowledge. In practice we often have extra information about the problem at hand, which could and should be used to guide the forecasting. One can incorporate it by explicating all our prior knowledge $z$, and place it on an extra input tape of our universal Turing machine $U$, or prefix our observation sequence $x$ by $z$ and use $M(z x)$ for prediction.

Another concern is the dependence of $K$ and $M$ on $U$. The good news is that a change of $U$ changes $K(x)$ only within an additive and $M(x)$ within a multiplicative constant independent of $x$. This makes the theory practically immune to any "reasonable" choice of $U$ for large data sets $x$, but predictions for short sequences (shorter than typical compiler lengths) can be arbitrary. One solution is to take into account our (whole) scientific prior knowledge $z$ Hut06, and predicting the now long string $z x$ leads to good (less sensitive to "reasonable" $U$ ) predictions. This is a kind of grand transfer learning scheme. It is unclear whether a more elegant theoretical solution is possible.

Finally, the incomputability of $K$ and $M$ prevents a direct implementation of Solomonoff induction. Most fundamental theories have to be approximated for practical use, sometimes systematically like polynomial time approximation algorithms or numerical integration, and sometimes heuristically like in many AI-search problems or in non-convex optimization problems. Universal machine learning is similar, except that its core quantities are only semi-computable. This makes them often hard, but as described in the previous section, not impossible, to approximate.

In any case, universal induction can serve as a "gold standard" which practitioners can aim at. Solomonoff's theory considers the class of all computable (stochastic) models, and a universal prior inspired by Ockham and Epicurus, quantified by Kolmogorov complexity. This lead to a universal theory of induction, prediction, decisions, and, by including Bellman, to universal actions in reactive environments.

Future progress on the issues above (incorporating prior knowledge, getting rid of the compiler constants, and finding better approximations) will lead to new insights and will continually increase the number of applications. 


\section{Recommended Reading}

[CV05] R. Cilibrasi and P. M. B. Vitányi. Clustering by compression. IEEE Trans. Information Theory, 51(4):1523-1545, 2005.

[Gag07] M. Gaglio. Universal search. Scholarpedia, 2(11):2575, 2007.

[Grü07] P. D. Grünwald. The Minimum Description Length Principle. The MIT Press, Cambridge, 2007.

[Hut05] M. Hutter. Universal Artificial Intelligence: Sequential Decisions based on Algorithmic Probability. Springer, Berlin, 2005.

[Hut06] M. Hutter. Human knowledge compression prize, 2006. open ended, http://prize.hutter1.net/.

[Hut07] M. Hutter. On universal prediction and Bayesian confirmation. Theoretical Computer Science, 384(1):33-48, 2007.

[LV08] M. Li and P. M. B. Vitányi. An Introduction to Kolmogorov Complexity and its Applications. Springer, Berlin, 3rd edition, 2008.

[Sch02] J. Schmidhuber. Hierarchies of generalized Kolmogorov complexities and nonenumerable universal measures computable in the limit. International Journal of Foundations of Computer Science, 13(4):587-612, 2002.

[Sol64] R. J. Solomonoff. A formal theory of inductive inference: Parts 1 and 2. Information and Control, 7:1-22 and 224-254, 1964.

[Sol78] R. J. Solomonoff. Complexity-based induction systems: Comparisons and convergence theorems. IEEE Transactions on Information Theory, IT-24:422-432, 1978.

[VNHS10] J. Veness, K. S. Ng, M. Hutter, and D. Silver. Reinforcement learning via AIXI approximation. In Proc. 24th AAAI Conference on Artificial Intelligence, Atlanta, 2010. AAAI Press.

[WM97] D. H. Wolpert and W. G. Macready. No free lunch theorems for optimization. IEEE Transactions on Evolutionary Computation, 1(1):67-82, 1997.

[ZL70] A. K. Zvonkin and L. A. Levin. The complexity of finite objects and the development of the concepts of information and randomness by means of the theory of algorithms. Russian Mathematical Surveys, 25(6):83-124, 1970. 